http://jmscr.igmpublication.org/home/ ISSN (e)-2347-176x ISSN (p) 2455-0450 crossref DOI: https://dx.doi.org/10.18535/jmscr/v8i9.28

\title{
Does Presoaking of Autografts in Vancomycin Reduces the risk of Infection After ACL Reconstruction (?): A Retrospective Study
}

\author{
Authors \\ Bijayendra Nath Chaturvedi $^{1}$, Omprakash Bhimwal ${ }^{2}$, Shikha Dhaundiyal $^{3}$, \\ Davinder Singh ${ }^{4}$ \\ ${ }^{1}$ Senior Resident, Sports Injury Center (SIC, Ortho), VMMC \& Safdarjung Hospital, New Delhi. \\ ${ }^{2}$ Senior Resident, Sports Injury Center (SIC, Ortho), VMMC \& Safdarjung Hospital, New Delhi. \\ ${ }^{3}$ Senior Resident, Sports Injury Center (SIC, Ortho), VMMC \& Safdarjung Hospital, New Delhi. \\ ${ }^{4}$ Professor, Sports Injury Center (SIC, Ortho), VMMC \& Safdarjung Hospital, New Delhi \\ Corresponding Author
}

Dr Bijayendra Nath Chaturvedi

Senior Resident (Orthopaedics), Sports Injury Center (SIC, Ortho), VMMC \& SafdarjunG Hospital,

New Delhi, India

\begin{abstract}
Background: infections in ACL reconstruction surgery varies from frank septic arthritis to mild discharge at site of tibial tunnel. Since it is devastating complication both for patient as well as surgeon, and autografts are a frequent source of infection. To prevent this complication, a retrospective study was carried out by pre-soaking of autografts in Vancomycin to reduce the risk of infection.

Purpose: Purpose of this study is to determine whether the presoaking of an anterior cruciate ligament (ACL) autograft in Vancomycin reduces the rate of infection following an ACL reconstruction or not.

Materials and Methods: A retrospective study of 1836 patients that consecutively underwent primary arthroscopic ACL Reconstructions with four stranded single bundle Hamstrings autograft over four years at our institute was performed. In the initial 2 years period, the patients 963 patients received preoperative IV antibiotics (group 1). In the following 2 years period, 873 patients received preoperative $I V$ antibiotics along with the graft was presoaked in a vancomycin solution (group 2). Presoaking was performed with sterile gauze previously saturated with a vancomycin solution $(5 \mathrm{mg} / \mathrm{ml})$.

Results: There were 963 and 873 patients in group 1 and 2, respectively. Eighteen cases of joint infections were identified in the series (0.98\%). All of these infections occurred in group 1, representing a rate of infection of $1.86 \%$ in comparison with $0 \%$ in group $2(p<0.001)$.

Conclusions: Presoaking of autograft with vancomycin in combination with perioperative intravenous antibiotic prophylaxis reduced the rate of knee joint infection following an ACL Reconstruction in comparison with perioperative i.v antibiotic prophylaxis alone. This technique could be of relevance in daily clinical practice to prevent infection after ACLR.

Study Design: Case control \& Retrospective comparative study; Level of evidence, 3.

Keywords: Vancomycin, Anterior cruciate ligament, pre-soaking, septic arthritis, Antibiotic prophylaxis, Biofilm.
\end{abstract}




\section{Introduction}

After Anterior Cruciate Ligament Reconstruction, infection is a rare but very devastating complication during early follow-up and autografts are the source of infection. most of the times with the incidence rate ranging from 0.14 to $1.87 \%{ }^{[1,2,3]}$. The best graft for $\mathrm{ACl}$ reconstruction is "Bone- patellar tendon-bone" (BPTB) graft as it consists of tendon and bony attachments. Though BPTB graft is considered to be as gold standard. But now a days Hamstrings tendons are in use for ACL reconstruction due to less graft harvesting site morbidity and easy technique. Strength of four strands single bundle Hamstrings Autograft is camparable to BPTB graft. In our study only cases of ACL reconstruction with Hamstrings autograft was included. The use of hamstring autograft, concomitant open surgical procedures, drains, and previous surgery on the same knee have been related to a higher risk of this complication $^{[4,5,6,7,8,9,10]}$.

Most important causative agents in up to $95 \%$ of cases are Staphylococci and among half of those are due to coagulase-negative staphylococci $(\mathrm{CNS})^{[1,6,7,11,12,13,14]}$. Other pathogens reported are Propionibacterium acnes and Enterobacter species $^{[1]}$. Some authors have linked this high percentage of CNS to the graft harvest and its contamination with patients' skin bacteria ${ }^{[1]}$.The use of a preoperative prophylactic intravenous antibiotic $^{[15]}$ as well as a proper hair removal, in the cases which needed $\mathrm{it}^{[16]}$, has demonstrated efficiency in reducing the rate of infection in orthopaedic procedures. Recently, autograft presoaking with vancomycin has been described as showing promising results in decreasing the rate of infection following an ACL Reconstruction ${ }^{[25]}$, although they only used hamstrings grafts in their studies. Administration of prophylactic intravenous antibiotics in knee surgery include at least 10 minutes before tourniquet inflation, 15 to 120 minutes before skin incision, or 30 to 60 minutes before the start of the procedure ${ }^{[18,19]}$.

$\mathrm{Pe}^{\prime}$ rez-Prieto et $\mathrm{al}^{[20]}$ published a study with the idea of identifying the point during the graft harvest and preparation process that the graft could be contaminated. Pe'rez-Prieto et $\mathrm{al}^{[20\}}$ identified the point during the graft harvest and preparation process that the graft could be contaminated. They took samples of the graft during harvesting and preparation with this purpose were obtained. Their results showed 7 cases $(14 \%)$ of graft contamination. In 5 cases $(10 \%)$, the graft was contaminated during preparation, and in 2 cases (4\%), the graft was contaminated during harvesting. Using the rationale that a contaminated graft could be the main cause of postoperative septic arthritis and in an effort to maximize the antibiotic efficacy of the graft, Vertullo et al ${ }^{[21]}$ described the "vancomycin wrap" technique in 2012. The practice involves wrapping a swab around the ACL graft before placement onto a tray or into a sterile plastic bag, at which point vancomycin solution is added, thus soaking the swab. Their first study showed a decrease in the infection rate in ACL reconstruction from $1.4 \%$ to $0 \%$. In the next years, a number of studies using the same protocol have appeared, all of them confirming the results of Vertullo and colleagues ${ }^{[22,23,24]}$.

The rationale for the use of vancomycin lies in its pharmacokinetic properties, which make it an ideal agent ${ }^{[25]}$. These include low allergenicity, heat stability, safety for local use, and large volume of distribution. It has a bactericidal action against skin commensals such as Staphylococcus aureus and coagulase-negative staphylococci, which are by far the most common pathogens isolated in ACL reconstruction infection ${ }^{[20]}$.

In a study published by Torres-Claramunt ${ }^{[13]}$, the rate of infection in the patients operated on for an ACL Reconstruction was assessed. This rate (1.8 $\%)$ was higher than initially expected. Therefore, presoaking of the autograft with vancomycin was introduced as a new prophylactic measure in the ACLR in order to reduce this rate of infection. The purpose of this study was to find out whether the implementation of prophylactic graft saturation with vancomycin reduces the rate of 
infection following an Anterior cruciate ligament Reconstruction, using Hamstrings Autograft.

\section{Material and Methods}

A retrospective review of all the patients that consecutively underwent primary arthroscopic ACLR with an Hamstrings autograft inour institutions. Those patients who needed an extraarticular procedure or those who received other types of autografts except Hamstrings or an allograft were not included in this study. In the initial 2 years period, the patients received preoperative IV antibiotics (group 1). In the following 2 years period, the patients received preoperative IV antibiotics along with the graft was presoaked in a vancomycin solution (group 2). Presoaking was performed with sterile gauze previously saturated with a vancomycin solution $(5 \mathrm{mg} / \mathrm{ml})$ as described by Vertullo et al

\section{Surgical Technique}

All patients were operated on by the same surgical team composed of four senior surgeons. All operations were performed on an outpatient basis. The prophylactic antibiotic protocol consisted in a single dose of $2 \mathrm{~g}$ of preoperative IV cefazolin or a single dose of $1 \mathrm{~g}$ of preoperative IV vancomycin, if a penicillin allergy was reported. No patient in this series informed of a vancomycin allergy. The types of graft employed were a quadrupled hamstrings Autograft. A pretension of $80 \mathrm{~N}$ was applied for $5 \mathrm{~min}$ before fixation of the hamstrings grafts. The hamstring grafts femoral fixation was carried out either with a transversal fixation (Cross-Pin system ${ }^{\circledR}$, Stryker) or with a cortical suspensory fixation system (XO Button Fixation System ${ }^{\circledR}$, ConMed Linvatec). Tibial fixation was accomplished with a resorbable interference screw. No drains were left after surgery. The technique of vancomycin saturation has been performed as previously described by Grayson et $\mathrm{al}^{[27]}$. A solution of $100 \mathrm{ml}$ of sterile saline was prepared in a tray and mixed with 500 $\mathrm{mg}$ of vancomycin powder. When the graft was obtained and prepared, it was immersed in the tray and then it was wrapped in gauze that had been saturated with this vancomycin solution beforehand. The graft was left there for 10-15 min (until it was used for the ACL Reconstruction).

\section{Diagnostic criteria for septic arthritis}

Diagnosis of septic arthritis was based on patient"s medical history and physical examination, laboratory parameters \{ESR. Crp\} and cultures of synovial fluid and/or joint tissue. \{Were there any discharge from tibial side \}Synovial aspiration was performed as soon as the diagnosis was suspected. The liquid obtained was immediately sent for biochemical analysis and cell count analysis in heparin tubes. The remaining synovial fluid was introduced into aerobic and anaerobic blood culture bottles for automatic colorimetric bacterial detection (BacT/ALERT® Culture Media). These bottles contain activated charcoal to neutralise antimicrobials and make for a more accurate diagnosis. They also make it possible to perform an antibiotic sensitivity test.

\section{Statistical Analysis}

Categorical variables were presented as means and range as percentages. When two related items of data were analysed, the Chi-square or Fisher exact test was used. In all cases, a $p$ value of $<0.05$ was considered statistically significant. A power analysis was performed to ensure an adequate sample size for the study. Selecting an incidence of infection of $1 \%$, a significance level $(\alpha)$ of 0.05 and a power $(\beta)$ of $80 \%$, the sample size needed was 119 patients in each group.

\section{Results}

There were 1836 patients who met the inclusion criteria. The first 963 were included in group 1, and the following 873 were included in group 2 . Both groups were similar in terms of age, sex and BMI (n.s.).

\section{Surgical data}

The type of graft employed was a quadrupled hamstring in $100 \%$ cases. There were 963 and 873 patients in group 1 and 2, respectively. Eighteen cases of joint infections were identified in the series $(0.98 \%)$. All of these infections occurred in 
group 1, representing a rate of infection of $1.86 \%$ in comparison with $0 \%$ in group $2(\mathrm{p}<0.001)$.

\section{Infection Case Analysis}

The bacteria isolated were as follows: 15 Staphylococcus species $(83.3 \%)$ of which 10 were CNS $(66.7 \%)$ and 5 Staphylococcus aureus (33.3\%); there was 2 cases of infections due to Propionibacterium acnes and one culture-negative infection. Six patients needed graft removal and a 2-stage ACL Reconstruction procedure because of treatment failure after the third or fourth arthroscopic debridement and lavage. \{ did culture was positive in all pts. Are there ant cases of strile culture\}

There was no infection among patients who received systemic antibiotic prophylaxis and graft presoaking with vancomycin (group 2). This represented a rate of infection of $0 \%$ ( 0 out of 873). Statistical analysis showed that the prior saturation of the ACL graft in a vancomycin solution significantly reduced the infection rate $(\mathrm{p}$ $<0.001)$ in comparison with patients in which this technique was not performed.

\section{Discussions}

Although knee joint infection following an ACLR is not as common as other implant-associated infections in orthopaedic surgery, the magnitude of this complication is equally important as an studies have been focused on the surgical management of this complication over recent years. Most of those studies concluded that aggressive arthroscopic debridement in combination with an antibiotic therapy should be the treatment of choice for this complication $[1,3,5,12,28]$. Despite this, antibiotic treatment guidelines remain unclear. Little has been reported about the prevention of a septic arthritis following an ACLR. Some proceedings such as antibiotic prophylaxis or limiting hair removal to the cases needing it, which are usually applied so as to avoid this complication, have been imported from other orthopaedic procedures (basically joint replacement procedures $)^{[15,16]}$. Grayson et al. ${ }^{[27]}$ studied the amount of vancomycin released from the pre-soaked bovine tendons. They observed that tendons elute vancomycin into the environment in which they were placed and where they act as a reservoir of vancomycin. Furthermore, tendons with larger dimensions provide a greater reservoir. This technique was posteriorly applied in an in vivo study by them. It showed an important decrease of the rate of joint infection following an ACLR when this prophylactic measure was included in the surgical protocol $^{[17]}$. Interestingly, their results were comparable to the findings observed in the present investigation. Vancomycin is a useful bactericidal drug against staphylococci and enterococci ${ }^{[24]}$ and has been described as an alternative in the treatment of Propionibacterium acnes implantassociated infections ${ }^{[29]}$. Vancomycin has shown to be safe for a local use ${ }^{[30]}$, and it has already been used in both local prophylaxis and treatment in orthopaedics. For instance, it has been utilised with antibiotic-loaded cement spacers or nails ${ }^{[31]}$, bioactive glasses or composite biomaterials ${ }^{[30]}$. Furthermore, it is a thermostable antibiotic and less toxic to eukaryotic cells than cefazolin or aminoglycosides $^{[32]}$. The minimal vancomycin concentration to eradicate most of Staphylococcus infections is about $2 \mu \mathrm{g} / \mathrm{ml}$.Grayson et al. ${ }^{[27]}$ showed that this concentration can be maintained for at least $24 \mathrm{~h}$ when a tendon has been previously presoaked with vancomycin and this same elution was lower than the reported osteoblast and chondroblast toxicity concentrations $^{[32]}$. Different studies have demonstrated that the infection rate using hamstrings autograft is higher than when using BPTB autograft ${ }^{[4,6]}$. In the current study, the percentage of hamstring autografts used was superior in the group where the graft was presoaked. However, the infection rate was $0 \%$ in this group. This suggests that graft presoaking with vancomycin reduces the infection rate following an ACLR despite the graft selected. Contamination of the graft can occur during harvest or even when it is introduced into the knee through the arthroscopic portals ${ }^{[6]}$. This might 
explain why the infection rate in hamstring autografts is higher ${ }^{[6]}$ and mostly due to CNS (patient's skin flora around the portal). Plante et al. ${ }^{[33]}$ found contamination in $23 \%$ of hamstring autografts. Moreover, the fact that the steeping of the graft in vancomycin reduces the infection rate would give support to this theory, even though specific microbiological studies are needed to confirm this hypothesis. Different limitations can be attributed to this study. First of all, it had a retrospective design with a lack of randomization. On the other hand, the fact that some steps in the surgical technique changed during the studied period might be considered another bias. The location of the femoral tunnel moved from an almost over the top position to an anatomical location in recent years, and the fixation by means of a transversal fixation has been replaced by a cortical suspensory system. This change was made because a cortical suspensory fixation system has been shown to be safer when an anatomical femoral tunnel placement is being performed [34,35]. Regardless this variation in the fixation system in the femur, no data suggested that the decrease in the rate of infection after ACLR could be attributed to the change of fixation. Despite these limitations, the present technique will be of relevance in daily clinical practice to prevent infection after ACLR.

Other antibiotics like Gentamycin, Bacitracin with polymyxin B or Povidine-iodine can also be used for the presoaking of grafts. But a in vitro study warned that presoaking of graft for 15 minutes in Bacitracin with Polymyxin will not sterilise the 30 $\%$ of the grafts. Similarly $10 \%$ Providing -Iodine solution for 30 minutes will not sterilise the grafts infected with two Coagulase Negative staphylococci.

\section{Conclusion}

Autograft presoaking with vancomycin in combination with classical intravenous antibiotic prophylaxis reduces the rate of knee joint infection following an ACLR in comparison with antibiotic prophylaxis alone.

\section{References}

1. Cadet ER, Makhni EC, Mehran N, Schulz BM (2013) Management of septic arthritis following anterior cruciate ligament reconstruction: a review of current practices and recommendations. J Am Acad Orthop Surg 21(11):647-656

2. Judd D, Bottoni C, Kim D, Burke M, Hooker S (2006) Infections following arthroscopic anterior cruciate ligament reconstruction. Arthroscopy 22(4):375384

3. Mouzopoulos G, Fotopoulos VC, Tzurbakis M (2009) Septic knee arthritis following ACL reconstruction: a systematic review. Knee Surg Sports Traumatol Arthrosc 17(9):1033-1042

4. Barker JU, Drakos MC, Maak TG, Warren RF, Williams RJ 3rd, Allen AA (2009) Effect of graft selection on the incidence of postoperative infection in anterior cruciate ligament reconstruction. Am J Sports Med 38(2):281-286

5. Judd D, Bottoni C, Kim D, Burke M, Hooker S (2006) Infections following arthroscopic anterior cruciate ligament reconstruction. Arthroscopy 22(4):375384

6. Maletis GB, Inacio MC, Reynolds S, Desmond JL, Maletis MM, Funahashi TT (2013) Incidence of postoperative anterior cruciate ligament reconstruction infections: graft choice makes a difference. Am J Sports Med 41(8):1780-1785

7. Sonnery-Cottet B, Archbold P, Zayni R, Bortolletto J, Thaunat M, Prost T, Padua VB, Chambat P (2011) Prevalence of septic arthritis after anterior cruciate ligament reconstruction among professional athletes. Am J Sports Med 39(11):2371-2376

8. Wang C, Lee YH, Siebold R (2014) Recommendations for the management of septic arthritis after ACL reconstruction. 
Knee Surg Sports Traumatol Arthrosc 22(9):2136-2144

9. Armstrong RW, Bolding F, Joseph R (1992) Septic arthritis following arthroscopy: clinical syndromes and analysis of risk factors. Arthroscopy $8(2): 213-223$

10. Schollin-Borg M, Michaelsson K, Rahme H (2003) Presentation, outcome, and cause of septic arthritis after anterior cruciate ligament reconstruction: a case control study. Arthroscopy 19(9):941-947

11. Kim YM, Joo YB (2012) Clinical presentation of staphylococcus epidermidis septic arthritis following anterior cruciate ligament reconstruction. Knee Surg Relat Res 24(1):46-51

12. Sonnery-Cottet B, Thaunat M, Archbold P, Issartel B, Cadet ER (2014) Management of septic arthritis following anterior cruciate ligament reconstruction: a review of current practices and recommendations. J Am Acad Orthop Surg 22(5):271-273

13. Torres-Claramunt R, Pelfort X, Erquicia J, Gil-Gonzalez S, Gelber PE, Puig L, Monllau JC (2012) Knee joint infection after ACL reconstruction: prevalence, management and functional outcomes. Knee Surg Sports Traumatol Arthrosc 21(12):2844-2849

14. Wang C, Lee YH, Siebold R (2014) Recommendations for the management of septic arthritis after ACL reconstruction. Knee Surg Sports Traumatol Arthrosc 22(9):2136-2144

15. Trampuz A, Zimmerli W (2006) Antimicrobial agents in orthopaedic surgery: prophylaxis and treatment. Drugs 66(8):1089-1105

16. Tanner J, Norrie P, Melen K (2011) Preoperative hair removal to reduce surgical site infection. Cochrane Database Syst Rev 9(11):CD004122

17. Vertullo CJ, Quick M, Jones A, Grayson JE (2013) A surgical technique using presoaked vancomycin hamstring grafts to decrease the risk of infection after anterior cruciate ligament reconstruction. Arthroscopy 28(3):337-342

18. Dhammi IK, Ul Haq R, Kumar S. Prophylactic antibiotics in orthopedic surgery: controversial issues in its use. Indian J Orthop. 2015;49(4): 373-376.

19. Jefferies JG, Aithie JMS, Spencer SJ. Vancomycin-soaked wrapping of harvested hamstring tendons during anterior cruciate ligament reconstruction: a review of the "vancomycin wrap." Knee. 2019; 26(3):524-529.

20. Pe 'rez-Prieto D, Portillo ME, TorresClaramunt R, Pelfort X, Hinarejos P, Monllau JC. Contamination occurs during ACL graft harvesting and manipulation, but it can be easily eradicated. Knee Surg Sports Traumatol Arthrosc. 2018;26 (2): 558-562.

21. Vertullo CJ, Quick M, Jones A, Grayson JE. A surgical technique using presoaked vancomycin hamstring grafts to decrease the risk of infection after anterior cruciate ligament reconstruction. Arthroscopy. 2012;28(3):337-342.

22. Naendrup JH, Marche B, de Sa D, et al. Vancomycin-soaking of the graft reduces the incidence of septic arthritis following ACL reconstruction: results of a systematic review and meta-analysis [published online January 17, 2019]. Knee Surg Sports Traumatol Arthrosc. doi: 10.1007/s00167-019-05353-1

23. Offerhaus C, Balke M, Hente J, Gehling M, Blendl S, Ho "her J.Vancomycin presoaking of the graft reduces postoperative infection rate without increasing risk of graft failure and arthrofibrosis in ACL reconstruction. Knee Surg Sports Traumatol Arthrosc. 2019;27(9):30143021.

24. Pe 'rez-Prieto $\mathrm{D}$, Torres-Claramunt $\mathrm{R}$, Gelber PE, Shehata TM, Pelfort X, 
Monllau JC. Autograft soaking in vancomycin reduces the risk of infection after anterior cruciate ligament reconstruction. Knee Surg Sports Traumatol Arthrosc. 2016;24(9):27242728.

25. Grayson JE, Grant GD, Dukie S, Vertullo CJ. The in vitro elution characteristics of vancomycin from tendons. Clin Orthop Relat Res. 2011; 469(10):2948-2952.

26. Viola R, Marzano N, Vianello R. An unusual epidemic of Staphylococcus negative infections involving anterior cruciate ligament reconstruction with salvage of the graft and function. Arthroscopy. 2000;16(2): 173-177

27. Grayson JE, Grant GD, Dukie S, Vertullo CJ (2011) The in vitro elution characteristics of vancomycin from tendons. Clin Orthop Relat Res 469(10):2948-2952

28. Abdel-Aziz A, Radwan YA, Rizk A (2013) Multiple arthroscopic debridement and graft retention in septic knee arthritis after ACL reconstruction: a prospective case-control study. Int Orthop 38(1):73-82

29. Portillo ME, Corvec S, Borens O, Trampuz A (2013) Propionibacterium acnes: an underestimated pathogen in implant-associated infections. Biomed Res Int 2013:804391

30. Hanssen AD (2005) Local antibiotic delivery vehicles in the treatment of musculoskeletal infection. Clin Orthop Relat Res 437:91-96

31. Wasko MK, Borens O (2013) Antibiotic cement nail for the treatment of posttraumatic intramedullary infections of the tibia: midterm results in 10 cases. Injury 44(8):1057-1060.
32. Edin ML, Miclau T, Lester GE, Lindsey RW, Dahners LE (1996) Effect of cefazolin and vancomycin on osteoblasts in vitro. Clin Orthop Relat Res 333:245251

33. Plante MJ, Li X, Scully G, Brown MA, Busconi BD, DeAngelis NA (2013) Evaluation of sterilization methods following contamination of hamstring autograft during anterior cruciate ligament reconstruction. Knee Surg Sports Traumatol Arthrosc 21(3):696-701

34. Gelber PE, Reina F, Torres R, Monllau JC (2010) Effect of femoral tunnel length on the safety of anterior cruciate ligament graft fixation using cross-pin technique: a cadaveric study. Am J Sports Med 38(9):1877-1884

35. Gelber PE, Reina F, Torres R, Monllau JC (2010) Effect of femoral tunnel length on the safety of anterior cruciate ligament graft fixation using cross-pin technique: a cadaveric study. Am J Sports Med 38(9):1877-1884. 